\title{
OTIMIZAÇÃO DO PROCESSO FOTOCATALÍTICO HETEROGÊNEO PARA O TRATAMENTO DE PERCOLADO DE ATERRO SANITÁRIO
}

\author{
Núbia Natália de Brito ${ }^{1,2}$, Giovani Archanjo Brota ${ }^{2}$, Ronaldo Teixeira Pelegrini ${ }^{3}$ e José Euclides Stipp Paterniani ${ }^{2}$ \\ 1- Instituto de Química IQ - Universidade Federal de Goiás (UFG). Rodovia Campus Samambaia, CP 131 CEP $74001-970$ - Goiânia - \\ Goiás, e-mail: nubiabrito@quimica.ufg.br \\ 2- Faculdade de Engenharia Agrícola- Universidade Estadual de Campinas (UNICAMP). Cidade Universitária Zeferino Vaz, C. P. 6011 \\ CEP:13083- 875 Campinas-SP \\ 3-Centro de Ciências Agrária CCA- Universidade Federal de São Carlos (UFSCAR).Rodovia Anhanguera Km17- SP 330, CEP: 13600- \\ 970 Araras-SP.
}

The conventional methods used in the treatment of this residue lead to a discharge with intense coloration, high organic matter concentration, toxic metals and high toxicity. This work had as objective to study a system integrated for the remediation of the leachate originating from the sanitary landfill of Limeira-SP city The system consisted in a preliminary treatment by a system of slow sand filtration and the subsequent treatment through advanced oxidative processes, considering that the heterogeneous photocatalysis technique with $\mathrm{TiO}_{2} /$ UV was experienced in remediation. The photocatalytic treatment was accomplished in a Pyrex glass reactor of approximately $1.7 \mathrm{~L}$ (100 $\mathrm{mm}$ of internal diameter, $145 \mathrm{~mm}$ of external diameter and total height of $300 \mathrm{~mm}$ ). The experiments were carried out through water refrigeration and internal recirculation system of the leachate. To optimize the treatment process the best methodology it was evaluated to minimize the coloration of the leachate, one of the parameters of larger removal difficulty. The results were satisfactory with leachate coloration reduction in the order of: $66 \%$ in $180 \mathrm{~min}$ and $76 \%$ in $540 \mathrm{~min}$ of treatment with the heterogeneous fotocatálise, using 700 mg.L $\mathrm{L}^{-1}$ of $\mathrm{TiO}_{2}, 45^{\circ} \mathrm{C}$ temperature, $15 \mathrm{~L} \cdot \mathrm{min}^{-1}$ air flow and 400 Watts lamp.

Keywords: Leachate, Sanitary Landfill, Slow Filtration, Photocatalysis.

\section{Introdução}

Os processos de tratamentos dos percolados de aterro foram iniciados a partir dos processos de tratamento de esgoto doméstico. Como esse resíduo é bem diferente os processos convencionais nem sempre respondem com a mesma eficiência obtida nos efluentes domésticos.

O tratamento químico através de Processos Oxidativos Avançados (POA) é uma técnica capaz de promover a degradação da matéria poluente e representa uma das tecnologias mais promissoras para tratamento de águas residuárias. $\mathrm{O}$ percolado quando tratado por POA como metodologia terciária pode promover uma depuração bastante significativa. Também podem ser utilizadas como pré-tratamento favorecendo a biodegradação. Esse manejo é mais indicado quando o percolado é originário de aterros "velhos" por possuírem macromoléculas orgânicas resistentes à degradação microbiológica [1].

Pesquisas recentes têm apontado para $\mathrm{o}$ emprego de processos combinados, fazendo uso das vantagens de diferentes métodos. Os tratamentos químicos como, por exemplo, os POA podem ser utilizados em conjunto com tratamentos biológicos para aumentar a biodegradabilidade de compostos recalcitrantes, diminuindo o tempo de tratamento dos tradicionais processos biológicos [1].

\section{Fotocatálise heterogênea}

A Fotocatálise Heterogênea é uma técnica capaz de gerar uma espécie química denominada de radical hidroxila $(\cdot \mathrm{OH})$. O radical hidroxila é um potente reagente químico com elevada capacidade de degradar praticamente todo tipo de poluente orgânico porque seu potencial de oxidação é muito elevado $\left(\mathrm{E}^{\circ}=+2,8\right.$ $\mathrm{V})$, podendo reagir com quase todo tipo de substância, 
subtraindo dela elétrons ou átomos de hidrogênio ou ainda adicionando-se às duplas ligações, tornando esta metodologia de grande interesse para uso em recuperação de ambientes quimicamente degradados [2].

$\mathrm{O} \mathrm{TiO}_{2}$ é o semicondutor mais utilizado na Fotocatálise Heterogênea devido às várias propriedades interessantes tais como: possibilidade de ativação por luz, apresenta insolubilidade em água, estabilidade química numa ampla faixa de $\mathrm{pH}$, possibilidade de imobilização em sólidos, baixo custo, possibilidade de reutilização e ausência de toxicidade [2].

O processo da excitação fotocatalítica do $\mathrm{TiO}_{2}$ demanda uma fonte luminosa (geralmente UV) e presença de oxigênio que é usado para retirar os elétrons do semicondutor foto-sensibilizado e favorecer a geração do radical hidroxila (eq. 1) [3].

$$
\begin{aligned}
& \mathrm{TiO}_{2}+\mathrm{H}_{2} \mathrm{O}+\mathrm{O}_{2}+\mathrm{UV}{ }^{\circledR} \mathrm{OH}+ \\
& \mathrm{O}_{2} \cdot-\mathrm{H}^{+}+\mathrm{TiO}_{2}
\end{aligned}
$$

\section{MATERIAL E METODOS}

Amostras de chorume foram obtidas de um aterro sanitário municipal com mais de 14 anos de idade localizado em Limeira (São Paulo, Brasil). Este aterro recebe resíduos sólidos municipais e resíduos industriais classe I e IIA e IIB. As amostras foram coletadas em garrafas de polipropileno e mantidas a $4^{\circ} \mathrm{C}$, protegidas da luz. Algumas características físico-químicas do chorume estão apresentadas na Tabela 1 .

\begin{tabular}{|c|c|}
\hline Parâmetros & Chorume in natura \\
\hline Nitrogênio $\mathrm{NH}_{4}^{+}\left(\mathrm{mg} \mathrm{L}^{-1}\right)$ & 446,67 \\
\hline Nitrito $\mathrm{NO}_{2}^{-}\left(\mathrm{mg} \mathrm{L}^{-1}\right)$ & 0,22 \\
\hline Nitrato $\mathrm{NO}_{2}^{-}\left(\mathrm{mg} \mathrm{L}^{-1}\right)$ & 2,66 \\
\hline Cloretos $\left(\mathrm{mg} \mathrm{L}^{-1}\right)$ & 1825 \\
\hline Ferro $\left(\mathrm{mg} \mathrm{L}^{-1}\right)$ & 54,16 \\
\hline $\mathrm{pH}$ & 8,01 \\
\hline Cor (Abs $400 \mathrm{~nm})$ & 2,460 \\
\hline Turbidez UNT & 68,7 \\
\hline Condutividade $\left(\mathrm{mS} \mathrm{cm}^{-1}\right)$ & 12,21 \\
\hline Fenóis Totais $\left(\mathrm{mg} \mathrm{L}^{-1}\right)$ & 24,09 \\
\hline $\operatorname{COT}\left(\mathrm{mg} \mathrm{L}^{-1}\right)$ & 3029,54 \\
\hline $\operatorname{AOX}\left(\mathrm{mg} \mathrm{L}^{-1}\right)$ & 0,905 \\
\hline
\end{tabular}

Tabela 1 - Caracterização Físico-química do Chorume

O semicondutor utilizado no processo fotocatalítico heterogêneo Dióxido de Titânio - Degussa P25 $\left(\mathrm{TiO}_{2}\right)$ foi adquirido da indústria Degussa S.A. na cidade de São Paulo.

\section{Tratamento fotocatalítico}

O tratamento fotocatalítico foi realizado em um reator de vidro Pyrex com capacidade de 1,7 L (100 mm de diâmetro interno, $145 \mathrm{~mm}$ de diâmetro externo e altura total de $300 \mathrm{~mm}$ ) equipado com sistema de refrigeração à água e recirculação do chorume. Uma representação esquemática do reator pode ser visualizada na Figura 1.

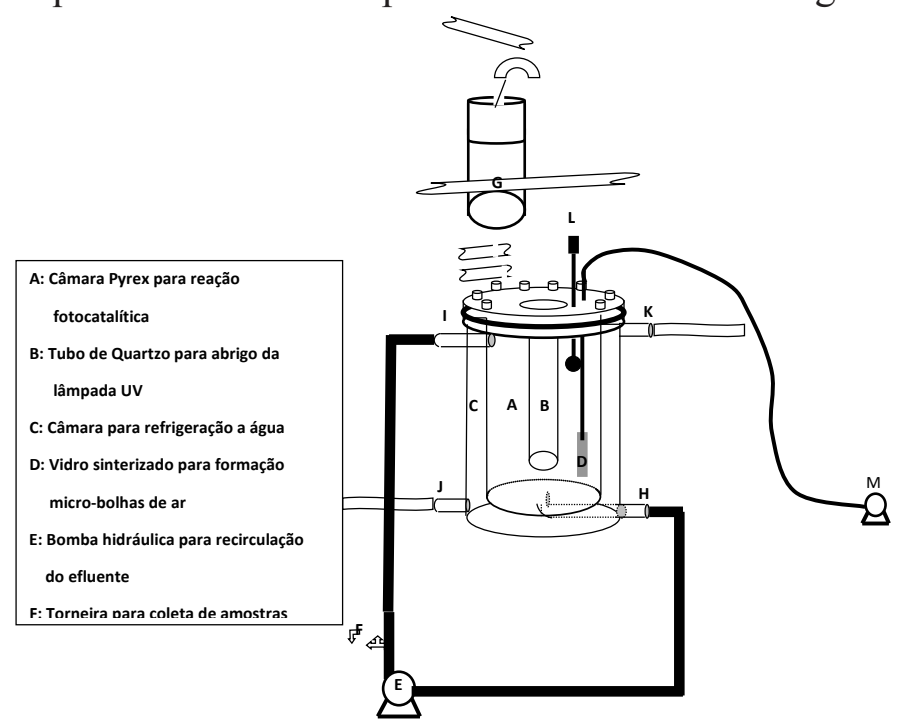

Figura 1- Esquema do reator fotocatalítico

A radiação UV foi provida por uma lâmpada de alta pressão de mercúrio (Philips HPL-N 250 e 400 W) com capacidade para emitir comprimentos de onda na região do UV pela remoção do bulbo exterior localizado no centro do reator.

Os experimentos foram processados utilizando refrigeração à água permitindo controlar a temperatura. A agitação do processo foi mantida contínua por meio da recirculação do percolado através de uma bomba hidráulica (Invensys Bav 1115-02U 220 V 60 Hz $34 \mathrm{~W})$. O fornecimento de oxigênio para o processo fotocatalítico foi mantido por borbulhamento de ar através de um vidro sinterizado localizado na parte inferior do reator utilizando para isso um compressor com válvula controladora de vazão (Inalar Compact).

A massa de $\mathrm{TiO}_{2}$ bem como o chorume a ser tratado era adicionada na parte superior do reator $(\mathrm{G})$. $\mathrm{O}$ dióxido de titânio era misturado por meio da recirculação do chorume. Para o controle analítico, alíquotas foram retiradas em intervalos convenientes e centrifugadas. 


\section{Resultados e Discussão}

\section{Estudo da otimização do processo fotocatalítico heterogêneo utilizando TiO_UV}

Para otimizar o tratamento fotocatalítico iniciou-se a partir de um planejamento fatorial $2^{3}$ (duas variáveis e três parâmetros) realizado por um período de 240 minutos, definido como tempo suficiente por meio de estudos preliminares de 12 horas, utilizando radiação ultravioleta através de uma lâmpada de 250 Watts. O percolado de resíduos pós tratamento por filtração lenta apresentou absorvância de 2,05 em comprimento de onda máximo de $400 \mathrm{~nm}$ (dentro da faixa do visível). O estudo avaliou dois pontos da concentração de dióxido de titânio de $900 \mathrm{mg} . \mathrm{L}^{-1}$ e $600 \mathrm{mg} . \mathrm{L}^{-1}$, vazão de ar de $15 \mathrm{~L}^{\mathrm{m}} \mathrm{min}^{-1}$ e 5 L.min ${ }^{-1}$ e temperatura de $40^{\circ} \mathrm{C}$ e $25^{\circ} \mathrm{C}$. Para avaliar a eficiência do tratamento foi analisada a melhor redução da cor por ser este parâmetro um fator limitante quando o percolado é tratado por processos convencionais (Tabela 2).

Tabela 2 - Otimização por Planejamento Fatorial $2^{3}$, do tratamento fotocatalítico do percolado de resíduos pós filtração lenta.

Condições: Lâmpada 250 Watts; Tempo 240 min.

\begin{tabular}{|c|c|c|c|c|}
\hline \multirow[t]{2}{*}{ Parâmetros } & \multicolumn{4}{|c|}{ Variáveis } \\
\hline & \multicolumn{3}{|c|}{$(+)$} & $(-)$ \\
\hline $\begin{array}{c}\text { Concentração de } \\
\text { TiO }_{2}\left(\mathrm{mg}^{-L^{-1}}\right)\end{array}$ & \multicolumn{3}{|c|}{900} & 600 \\
\hline $\begin{array}{c}\text { Vazão } \\
\left(\text { L.min }^{-1}\right)\end{array}$ & \multicolumn{3}{|c|}{15} & 5 \\
\hline $\begin{array}{c}\text { Temperatura } \\
\left({ }^{\circ} \mathrm{C}\right)\end{array}$ & \multicolumn{3}{|c|}{40} & 25 \\
\hline Testes & TiO, & Ar & Temp. & $\begin{array}{c}\text { Red. Cor } \\
(\%)\end{array}$ \\
\hline 1 & + & + & + & 37,94 \\
\hline 2 & + & + & - & 31,56 \\
\hline 3 & + & - & + & 33,05 \\
\hline 4 & + & - & - & 12,01 \\
\hline 5 & - & + & + & 31,14 \\
\hline 6 & - & + & - & 32,41 \\
\hline 7 & - & - & + & 19,87 \\
\hline 8 & - & - & - & 26.46 \\
\hline
\end{tabular}

Importante ressaltar que o percolado foi tratado "in natura" sem nenhuma diluição, as diluições foram realizadas após tratamento para leitura no espectrofotômetro a fim de obedecer à lei de Beer.
Com os resultados obtidos por meio do planejamento fatorial (Tabela 2), observou-se que as condições do teste 1 (concentração de $\mathrm{TiO}_{2}$ de 900 mg.L.-1 ; vazão de ar 15 L.min ${ }^{-1}$ e temperatura de $40^{\circ} \mathrm{C}$ ) apresentou maior eficiência na redução da cor do percolado (37,94\%). A partir destes dados, iniciou-se um estudo mais detalhado com a concentração do $\mathrm{TiO}_{2}$ para verificar a redução da cor nas proximidades do valor encontrado no planejamento fatorial. Os resultados estão apresentados na Tabela 3.

No estudo de otimização da massa de $\mathrm{TiO}_{2}$ pôdese verificar que a concentração $700 \mathrm{mg} . \mathrm{L}^{-1}$ apresentou maior redução da coloração do percolado. A partir desta concentração observou-se uma pequena redução da eficiência do processo fotocatalítico indicando que o excesso de $\mathrm{TiO}_{2}$ deixa a solução mais turva impedindo a passagem da radiação ultravioleta prejudicando a fotocatálise.

Um estudo de "abertura" dos parâmetros vazão e temperatura, similar ao realizado com a concentração de $\mathrm{TiO}_{2}$, também foi realizado para verificar a máxima eficiência de redução da cor. Entretanto, a temperatura do sistema, que era mantida através da lâmpada, ficou limitada a no máximo $40^{\circ} \mathrm{C}$ em função da potência da lâmpada de 250 Watts.

Tabela 3 - Variação da massa de $\mathrm{TiO}_{2}$ em torno da concentração otimizada (900 mg. $\mathrm{L}^{-1}$ ) no planejamento fatorial. Condições: Lâmpada 250 W; Tempo 240 min; Vazão de ar 15 L.min $^{-1}$; Temperatura de $40^{\circ} \mathrm{C}$.

\begin{tabular}{c|c|c|c|c}
\hline \multirow{2}{*}{ Testes } & \multirow{2}{*}{$\begin{array}{c}\text { Concentração } \\
\text { de TiO } \\
\text { (mg. }\end{array}$} & \multicolumn{2}{|c|}{$\begin{array}{c}\text { Absorvância 400 nm } \\
\text { (fator de diluição 4) }\end{array}$} & \multirow{2}{*}{$\begin{array}{c}\text { Redução da } \\
\text { Cor (\%) }\end{array}$} \\
\cline { 3 - 4 } & & Antes & $\begin{array}{c}\text { Pós- } \\
\text { Fotoc }\end{array}$ & \\
\hline 1 & 600 & 0,480 & 0,299 & 37,71 \\
\hline 2 & 650 & 0,477 & 0,293 & 38,57 \\
\hline 3 & $\mathbf{7 0 0}$ & $\mathbf{0 , 4 7 8}$ & $\mathbf{0 , 2 7 7}$ & $\mathbf{4 2 , 0 5}$ \\
\hline 4 & 750 & 0,475 & 0,292 & 38,52 \\
\hline 5 & 800 & 0,482 & 0,299 & 37,96 \\
\hline 6 & 850 & 0,478 & 0,301 & 37,02 \\
\hline 7 & 900 & 0,476 & 0,295 & 38,02 \\
\hline 8 & 1000 & 0,472 & 0,304 & 35,59 \\
\hline 9 & 1200 & 0,474 & 0,319 & 32,70 \\
\hline 10 & 1400 & 0,475 & 0,317 & 33,26 \\
\hline
\end{tabular}

Temperaturas mais baixas eram alcançadas através do controle da entrada de água para refrigeração do reator fotocatalítico, sendo que, nenhum resultado conseguido com temperatura mais baixa foi superior ao alcançado com a temperatura de $40^{\circ} \mathrm{C}$ na redução da cor do percolado. 
A vazão de ar de 15 L.min ${ }^{-1}$, que apresentou melhor resultado no planejamento fatorial, representa a vazão máxima alcançada pela bomba de ar do sistema de tratamento fotocatalítico. Nesta vazão foi conseguido o maior valor de redução da coloração do percolado, indicando a importância do oxigênio na retirada do elétron na banda de condução do semicondutor.

Os estudos de otimização da fotocatálise, com a lâmpada de 250 Watts, prosseguiram-se a partir da ampliação do tempo de tratamento. Após a otimização dos parâmetros: concentração de $\mathrm{TiO}_{2}$ de $700 \mathrm{mg} \cdot \mathrm{L}^{-1}$; vazão de ar $15 \mathrm{~L} \cdot \mathrm{min}^{-1}$ e temperatura de $40^{\circ} \mathrm{C}$, o tempo de tratamento foi estendido até 720 minutos (12 horas) (Tabela 4).

A análise da tendência do tratamento referente aos dados da Tabela 3 pode ser visualizada na Figura 2.

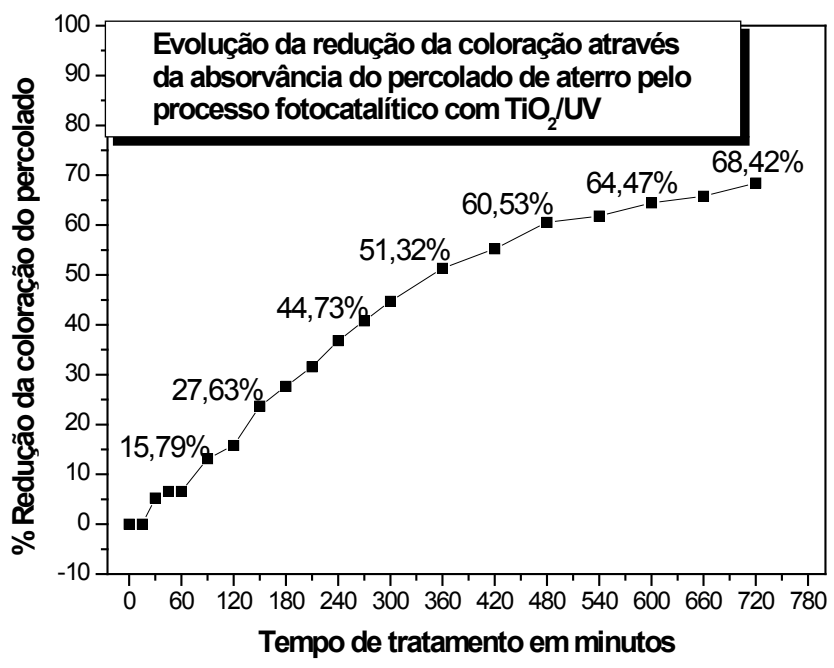

Figura 2 - Estudo da tendência do tratamento fotocatalítico através do tempo. Concentração de $\mathrm{TiO}_{2}$ de $700 \mathrm{mg} \cdot \mathrm{L}^{-1}$; Vazão de

ar 15 L.min ${ }^{-1}$; Temperatura de $40^{\circ} \mathrm{C}$; Lâmpada de $250 \mathrm{~W}$.

$\mathrm{Na}$ análise da tendência da curva da Figura 2, observa-se que o processo fotocatalítico nas condições estudadas está atingido seu limite podendo esperar pequenas reduções da coloração se o tempo for estendido acima de 12 horas. Pode-se observar também que a partir de 480 minutos ( 8 horas) de tratamento, ou seja, nas 4 horas finais, houve apenas uma redução da coloração em torno de $8 \%$, o que não justifica estender o tempo de tratamento em função da baixa eficiência e dos gastos com energia.
Tabela 4 - Estudo da variação do tempo para fotocatálise do percolado. Concentração de $\mathrm{TiO}_{2}$ de 700 mg.L.-1; Vazão de ar 15 L. min $^{-1}$; Temperatura de $40^{\circ} \mathrm{C}$; Lâmpada de $250 \mathrm{~W}$.

\begin{tabular}{c|c|c|c}
\hline Testes & $\begin{array}{c}\text { Tempo } \\
\text { (min) }\end{array}$ & $\begin{array}{c}\text { Absorvância 400 } \\
\mathbf{n m} \\
\text { (fator de diluição } \\
\mathbf{4})\end{array}$ & $\begin{array}{c}\text { Redução } \\
\text { da Cor } \\
\mathbf{( \% )}\end{array}$ \\
\hline 1 & 0 & 0,760 & \\
\hline 2 & 15 & 0,760 & $0,00 \%$ \\
\hline 3 & 30 & 0,720 & $5,26 \%$ \\
\hline 4 & 45 & 0,710 & $6,58 \%$ \\
\hline 5 & 60 & 0,710 & $6,58 \%$ \\
\hline 6 & 90 & 0,660 & $13,16 \%$ \\
\hline 7 & 120 & 0,640 & $15,79 \%$ \\
\hline 8 & 150 & 0,580 & $23,68 \%$ \\
\hline 9 & 180 & 0,550 & $27,63 \%$ \\
\hline 10 & 210 & 0,520 & $31,58 \%$ \\
\hline 11 & 240 & 0,480 & $36,84 \%$ \\
\hline 12 & 270 & 0,450 & $40,79 \%$ \\
\hline 13 & 300 & 0,420 & $44,73 \%$ \\
\hline 14 & 360 & 0,370 & $51,32 \%$ \\
\hline 15 & 420 & 0,340 & $55,26 \%$ \\
\hline 16 & 480 & 0,300 & $60,53 \%$ \\
\hline 17 & 540 & 0,290 & $61,84 \%$ \\
\hline 18 & 600 & 0,270 & $64,47 \%$ \\
\hline 19 & 660 & 0,260 & $65,79 \%$ \\
\hline $\mathbf{2 0}$ & $\mathbf{7 2 0}$ & $\mathbf{0 , 2 4 0}$ & $\mathbf{6 8 , 4 2 \%}$ \\
\hline & & &
\end{tabular}

Nesta primeira etapa de otimização pôde-se concluir que sob condições de: concentração de $\mathrm{TiO}_{2} 700$ mg.L $\mathrm{L}^{-1}$, vazão de ar de $15 \mathrm{~L} . \mathrm{min}^{-1}$, temperatura de $40^{\circ} \mathrm{C}$, lâmpada de 250 Watts e tempo de tratamento de 480 minutos (8 horas) a máxima porcentagem de redução da coloração do percolado encontrada foi de $60,53 \%$. Com o propósito de reduzir o tempo de tratamento fotocatalítico e aumentar eficiência na descoloração realizou-se uma nova otimização utilizando uma lâmpada com maior potência (400 Watts).

Para iniciar os estudos com a lâmpada de 400 Watts fixou-se o tempo de tratamento em 240 minutos (4 horas), a temperatura em $40^{\circ} \mathrm{C}$, a vazão de ar em 15 L.min ${ }^{-1}$ e variou-se a concentração de $\mathrm{TiO}_{2}$ (Tabela 5).

Similar aos resultados alcançados nos estudos de otimização com lâmpada de 250 Watts, ao utilizar uma lâmpada de 400 Watts à máxima redução da coloração foi encontrada utilizando a concentração de $700 \mathrm{mg} . \mathrm{L}^{-1}$ de dióxido de titânio, sendo observado uma melhor eficiência na redução da cor, em torno de $72,9 \%$ (Tabela 5).

Para melhor visualização dos resultados (Tabela 5), foi construída a Figura 3 onde podem ser observadas as absorvâncias em função das concentrações de $\mathrm{TiO}_{2}$ utilizadas no tratamento fotocatalítico do percolado. 


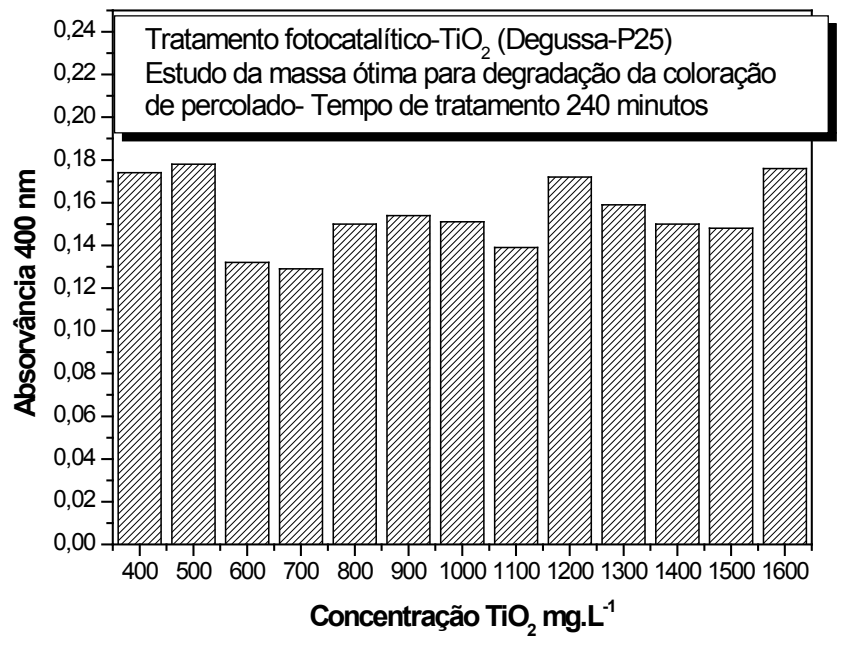

Figura 3 - Otimização da massa de $\mathrm{TiO}_{2}$ para estudo da descoloração do percolado de aterro empregando lâmpada de 400 W. Vazão de ar: 15 L. min $^{-1}$; Temperatura de 40ㅡ; Tempo de tratamento $240 \mathrm{~min}$.

Tabela 5 - Estudo fotocatalítico da concentração ótima de $\mathrm{TiO}_{2}$ para lâmpada de 400 Watts. Temperatura $40^{\circ} \mathrm{C}$; Vazão de $\operatorname{ar} 15^{2}$ L. $\mathrm{min}^{-1}$; Tempo de tratamento $240 \mathrm{~min}$.

\begin{tabular}{c|c|c|c|c}
\hline \multirow{2}{*}{ Testes } & Concentração & \multicolumn{2}{|c|}{$\begin{array}{c}\text { Absorvância 400 nm } \\
\text { (fator de diluição 4) }\end{array}$} & \multirow{2}{*}{$\begin{array}{c}\text { Redução } \\
\text { de TiO }\end{array}$} \\
\cline { 3 - 4 } & $\begin{array}{c}\text { Pós- } \\
\left.\text { (mg. } \mathbf{L}^{-1}\right)\end{array}$ & Antes & $\begin{array}{c}\text { Fotoc } \\
\text { (\%) }\end{array}$ \\
\hline 1 & 400 & 0,485 & 0,174 & 64,12 \\
\hline 2 & 500 & 0,477 & 0,178 & 62,68 \\
\hline 3 & 600 & 0,470 & 0,132 & 71,91 \\
\hline $\mathbf{4}$ & $\mathbf{7 0 0}$ & $\mathbf{0 , 4 7 6}$ & $\mathbf{0 , 1 2 9}$ & $\mathbf{7 2 , 9 0}$ \\
\hline 5 & 800 & 0,465 & 0,150 & 67,74 \\
\hline 6 & 900 & 0,468 & 0,154 & 67,09 \\
\hline 7 & 1000 & 0,461 & 0,151 & 67,25 \\
\hline 8 & 1100 & 0,477 & 0,139 & 70,86 \\
\hline 9 & 1200 & 0,472 & 0,172 & 63,56 \\
\hline 10 & 1300 & 0,472 & 0,159 & 66,31 \\
\hline 11 & 1400 & 0,472 & 0,150 & 68,22 \\
\hline 12 & 1500 & 0,474 & 0,148 & 68,78 \\
\hline 13 & 1600 & 0,474 & 0,176 & 62,87 \\
\hline
\end{tabular}

A partir da massa ótima do semicondutor $\mathrm{TiO}_{2}$ (concentração de 700 mg.L-1 ) vazão de ar em 15 L.min ${ }^{-1}$, foi realizado estudo da otimização da temperatura em testes de 240 minutos de tratamento fotocatalítico. A variação da temperatura foi de: $40^{\circ} \mathrm{C}, 45^{\circ} \mathrm{C}$ e $50^{\circ} \mathrm{C}$ (Tabela 6).
Tabela 6 - Otimização da temperatura para estudos fotocatalíticos de redução de cor de percolado. Lâmpada de 400 W; Tempo de tratamento 240 min; Vazão de ar 15 L. min-1; Concentração $\mathrm{TiO}_{2} 700 \mathrm{mg} \cdot \mathrm{L}^{-1}$.

\begin{tabular}{c|c|c|c|c}
\hline \multirow{2}{*}{ Testes } & \multirow{2}{*}{ Temperatura } & \multicolumn{2}{|c|}{$\begin{array}{c}\text { Absorvância } \\
\mathbf{4 0 0} \text { nm (fator de } \\
\text { diluição 4) }\end{array}$} & \multirow{2}{*}{$\begin{array}{c}\text { Redução da } \\
\text { Cor (\%) }\end{array}$} \\
\cline { 3 - 4 } & & Antes & $\begin{array}{c}\text { Pós- } \\
\text { Fotoc }\end{array}$ & \\
\hline 1 & $40^{\circ} \mathrm{C}$ & 0,390 & 0,176 & 54,87 \\
\hline 2 & $\mathbf{4 5}^{\circ} \mathrm{C}$ & $\mathbf{0 , 4 2 0}$ & $\mathbf{0 , 1 2 8}$ & $\mathbf{6 9 , 5 2}$ \\
\hline 3 & $50^{\circ} \mathrm{C}$ & 0,386 & 0,183 & 52,59 \\
\hline
\end{tabular}

Com a lâmpada de 400 Watts foi possível estender a temperatura acima de $40^{\circ} \mathrm{C}$, sendo que, temperaturas superiores a $50^{\circ} \mathrm{C}$ deixaram as conexões do reator (em polipropileno) em condições de risco para a operação do reator fotocatalítico. Das temperaturas estudadas a que apresentou maior eficiência na descoloração (medida em função da absorvância) do percolado foi a de $45^{\circ} \mathrm{C}$.

Em um processo fotocatalítico, quando a temperatura é aumentada de $23^{\circ} \mathrm{C}$ para $45^{\circ} \mathrm{C}$ pode ocasionar um efeito sinérgico na degradação da matéria orgânica, favorecendo a combinação com os radicais hidroxila $(\mathrm{OH})$ produzidos na fotocatálise [4].

A partir da massa ótima do semicondutor $\mathrm{TiO}_{2}$ (concentração de $700 \mathrm{mg} . \mathrm{L}^{-1}$ ) e temperatura de $45^{\circ} \mathrm{C}$, realizou-se o estudo da vazão ótima de ar. A variação estudada foi de: 5 L.min ${ }^{-1}, 10$ L.min ${ }^{-1}, 15$ L.min ${ }^{-1}$ (Tabela 7).

Tabela 7 - Otimização da vazão de ar para estudos fotocatalíticos de redução de cor do percolado. Lâmpada de 400 watts; Tempo de tratamento $240 \mathrm{~min}$; temperatura $45^{\circ} \mathrm{C}$; Concentração $\mathrm{TiO}_{2} 700$ mg. $L^{-1}$.

\begin{tabular}{|c|c|c|c|c|}
\hline \multirow[t]{2}{*}{ Testes } & \multirow{2}{*}{$\begin{array}{l}\text { Vazão de ar } \\
\left(\text { L. } \text { min }^{-1}\right)\end{array}$} & \multicolumn{2}{|c|}{$\begin{array}{l}\text { Absorvância } 400 \text { nm } \\
\text { (fator de diluição 4) }\end{array}$} & \multirow{2}{*}{$\begin{array}{c}\text { Redução } \\
\text { da Cor } \\
(\%) \\
\end{array}$} \\
\hline & & Antes & $\begin{array}{l}\text { Pós- } \\
\text { Fotoc }\end{array}$ & \\
\hline 1 & 5 & 0,390 & 0,176 & 56,76 \\
\hline 2 & 10 & 0,386 & 0,183 & 65,35 \\
\hline 3 & 15 & 0,420 & 0,128 & 69,52 \\
\hline
\end{tabular}

Para a otimização da vazão de ar o valor que apresentou maior eficiência na descoloração do percolado foi de $15 \mathrm{~L}$. $\mathrm{min}^{-1}$. Maior vazão de ar possibilita maior turbulência podendo aumentar o contato entre o oxigênio e $\mathrm{o} \mathrm{TiO}_{2}$ favorecendo a transferência de elétrons da banda de condução do semicondutor para o oxigênio beneficiando assim a fotocatálise [4].

A partir da massa ótima do semicondutor $\left(\mathrm{TiO}_{2}\right)$ na concentração de $700 \mathrm{mg} . \mathrm{L}^{-1}$, da vazão de 15 
L.min. ${ }^{-1}$ e da temperatura de $45^{\circ} \mathrm{C}$, foi realizado o estudo cinético do tempo de tratamento fotocatalítico durante 540 min (Tabela 8).

Tabela 8 - Estudo da variação do tempo para fotocatálise do percolado. Concentração de $\mathrm{TiO}_{2}$ de 700 mg.L-1 ; Vazão de ar 15 L. min $^{-1}$; Temperatura de $45^{\circ} \mathrm{C}$; Lâmpada de $400 \mathrm{~W}$.

\begin{tabular}{c|c|c|c}
\hline Testes & $\begin{array}{c}\text { Tempo } \\
\text { (min) }\end{array}$ & $\begin{array}{c}\text { Absorvância 400 nm } \\
\text { (fator de diluição 4) }\end{array}$ & $\begin{array}{c}\text { Redução da } \\
\text { Cor (\%) }\end{array}$ \\
\hline 1 & 0 & 0,420 & 0,00 \\
\hline 2 & 30 & 0,323 & 23,10 \\
\hline 3 & 60 & 0,248 & 40,95 \\
\hline 4 & 90 & 0,202 & 51,90 \\
\hline 5 & 120 & 0,187 & 55,48 \\
\hline 6 & 180 & 0,144 & 65,71 \\
\hline 7 & 240 & 0,128 & 69,52 \\
\hline 8 & 300 & 0,133 & 68,33 \\
\hline 9 & 360 & 0,127 & 69,76 \\
\hline 10 & 420 & 0,122 & 70,95 \\
\hline 11 & 480 & 0,111 & 73,57 \\
\hline 12 & 540 & 0,098 & 76,67 \\
\hline
\end{tabular}

Estendendo-se o tempo de tratamento com as condições otimizadas (Concentração de $\mathrm{TiO}_{2}$ de 700 mg.L $L^{-1}$; Vazão de ar 15 L. $\min ^{-1}$; Temperatura de $45^{\circ} \mathrm{C}$; Lâmpada de 400 Watts), observou-se reduções da cor do percolado na ordem de $76,67 \%$ conseguida em 540 minutos (9 horas) de fotocatálise. Este resultado é bem considerável em razão de se tratar de uma matriz com características recalcitrantes que apresenta uma intensa coloração, parâmetro de difícil remoção para os principais métodos convencionais [5].

A análise da tendência do tratamento referente aos dados da Tabela 8, pode ser visualizada na Figura 4.

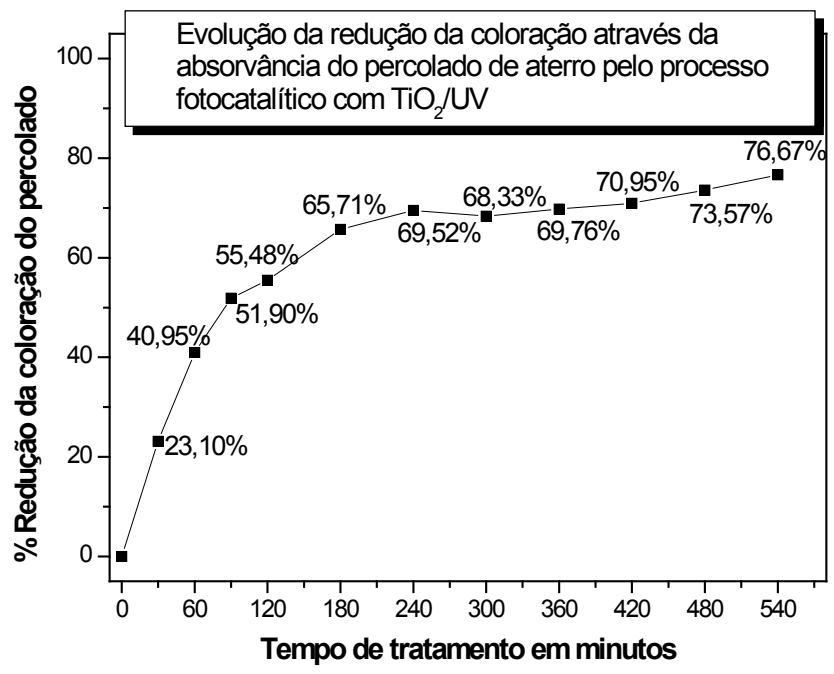

Figura 4- Evolução da descoloração do percolado empregando Tratamento Fotocatalítico. Concentração do semicondutor $\left(\mathrm{TiO}_{2}\right)$ 700 mg.L L $^{-1}$, Vazão de ar 15 L. $\mathrm{min}^{-1}$, Temperatura de $45^{\circ} \mathrm{C}$.
Embora extremamente eficientes, os POA apresentam duas limitações no tratamento de matrizes de percolado de aterros. Uma está representada pela elevada carga orgânica e a outra pela elevada concentração de ácidos húmicos, sendo assim a filtração lenta contribuiu na remediação do percolado para posterior tratamento fotocatalítico [5].

Pode-se concluir que este processo de otimização indicou que as condições que apresentaram melhores resultados foram: concentração de $\mathrm{TiO}_{2} 700$ mg.L. ${ }^{-1}$, vazão de ar de $15 \mathrm{~L} \cdot \mathrm{min}^{-1}$, temperatura de $45^{\circ} \mathrm{C}$, lâmpada de 400 Watts. O tempo de tratamento deve ficar a critério dos resultados que se queira alcançar.

\section{CONCLUSÃO}

As otimizações dos estudos sempre foram realizadas em função da diminuição da cor, um parâmetro de difícil remoção para os tratamentos convencionais. A fotocatálise heterogênea indicou que as condições que apresentaram melhores resultados foram: concentração de $\mathrm{TiO}_{2} 700 \mathrm{mg} . \mathrm{L}^{-1}$, vazão de ar de $15 \mathrm{~L}^{\mathrm{m}} \mathrm{min}^{-1}$, temperatura de $45^{\circ} \mathrm{C}$, lâmpada de 400 Watts. Nestas condições puderam-se observar reduções de $65,71 \%$ da coloração do percolado em 180 minutos de tratamento.

Após a conclusão deste estudo, pode-se verificar que, o percolado de aterro sanitário não deve e não pode ser considerado como um efluente doméstico, e, portanto, não pode ser remediado de maneira similar, porque apresenta características totalmente diferentes do ponto de vista físico, químico e biológico. Assim, o percolado apresenta-se como desafiador do ponto de vista de sua recuperação.

\section{AGRADECIMENTOS}

Os autores agradecem ao Conselho Nacional de Pesquisa Científica - $(\mathrm{CNPq})$ pelas bolsas concedidas e suporte técnico.

\section{REFERÊNCIAS BIBLIOGRÁFICAS}

[1] De Morais, J. L.; Peralta-Zamora, P. Use of advanced oxidation processes to improve the biodegradability of mature landfill leachates. Jour- 
nal of Hazardous Materials. 123 (1-3) (2005) 181.

[2] Nogueira, R.F.P.; Jardim, W.F. Heterogeneous photocatalysis and its environmental application. Química Nova. 21 (1) (1998) 69.

[3] Ziolli, R. L.; Jardim, W. F. Mechanism reactions of photodegradation of organic compounds catalyzed by TiO, Quimica Nova. 21(3) (1998) 319.

[4] Ferreira, I.V.L. Fotocatálise heterogênea com $\mathrm{TiO}_{2}$ aplicada ao tratamento de esgoto sanitário secundário. 160p. Tese (Doutorado em Hidráulica e Saneamento), Escola de Engenharia de São Carlos, Universidade de São Paulo. (2005).

[5] Pacheco, J.R.; Peralta-Zamora, P. Integração de processos físico-químicos e oxidativos avançados para remediação de percolado de aterro sanitário (chorume). Engenharia Sanitária e Ambiental. 9 (4) (2004) 306. 